\title{
Essais
}

ESSAIS

Revue interdisciplinaire d'Humanités

$1 \mid 2012$

Varia

\section{Invention d'un nouveau territoire de subjectivation comme évolution du bio-pouvoir dans les démocraties parlementaires}

Sandra Lemeilleur

\author{
(2) OpenEdition \\ Journals \\ Édition électronique \\ URL : https://journals.openedition.org/essais/11077 \\ DOI : 10.4000/essais. 11077 \\ ISSN : 2276-0970 \\ Éditeur \\ École doctorale Montaigne Humanités
}

Édition imprimée

Date de publication : 15 mai 2012

Pagination : 106-119

ISSN : 2417-4211

Référence électronique

Sandra Lemeilleur, «Invention d'un nouveau territoire de subjectivation comme évolution du biopouvoir dans les démocraties parlementaires », Essais [En ligne], 1 | 2012, mis en ligne le 31 janvier 2022, consulté le 02 février 2022. URL : http://journals.openedition.org/essais/11077 ; DOI : https:// doi.org/10.4000/essais. 11077 
Sandra Lemeilleur, Université Michel de Montaigne Bordeaux 3, EA 4426 MICA sandralemeilleur@yahoo.fr

\section{Résumé}

L'évolution de l'espace de vie suit l'évolution du concept d'intime. De la chambre individuelle à la colocation, la possibilité d'un espace à soi se réduit. L'invention des espaces du virtuel ainsi que les usages relatifs comme territoire de l'expression du sujet entretiennent des rapports étroits avec le régime politique des démocraties parlementaires. Ces liens permettent l'élaboration d'une nouvelle forme de subjectivité. L'intention de cet article est de montrer que l'intime est un concept interstitiel évoluant dans un espace interstitiel entre public et privé.

\section{Mot-clés}

intime, production de subjectivité, bio-pouvoir, virtuel, anthropologie des sciences del'information et dela communication

\section{Abstract}

The evolution of living space follows the evolution of the Intimate concept. From living in a single room to sharing a flat, the chance of having our own space is reduced. Virtual space invention and its related uses as a subject expressing territory maintain a close relationship with the political system of parliamentary democracies. These links allow the development of a new form of subjectivity. The intention of this paper is to show that Intimate is an interstitial concept operating in an interstitial space between Public and Private.

Key-words

Intimate, production of subjectivity, bio-power, virtual, anthropology of information and communication sciences 


\section{Invention d'un nouveau territoire de subjectivation comme évolution du bio-pouvoir dans les démocraties parlementaires}

La vie des individus semble mise à nu dans les espaces du virtuel. Pourquoi cette évolution? Depuis l'espace intime de la chambre, comment le sujet a-t-il conquis un nouvel espace - interstitiel - entre espace public et espace privé, entre espace physique et espace virtuel? Dire actuellement que les dispositifs de la Toile oppressent les individus à diffuser leur intimité sur des réseaux sociaux, des blogs ou - plus encore - sur des sites de dating ou de matchmaking est devenu un lieu commun. Comment condamner un objet alors qu'il est sans intentionnalité? Il s'agirait encore une fois de condamner les objets et de ne jamais se pencher sur les usages. Cette communication vise à interroger les usages de la production de subjectivité1. Le sujet ne se saisit-il pas aujourd'hui de ces dispositifs pour servir sa propre subjectivation : "Si je suis connecté, c'est que j'existe", dans la société démocratique actuelle? Comme l'analysait déjà Michel Foucault², pour l'Antiquité, le souci de soi est apparu suite à un affaiblissement du pouvoir politique entraînant un repli sur soi. De nos jours quels liens s'établissent entre le souci de soi et le système politique. Comment l'individu est-il passé dans l'espace public de citoyen à sujet utilisant son pouvoir d'actant dans le virtuel pour servir sa propre subjectivation? Comment la biopolitique a-t-elle entraîné la diffusion de la vie de chacun dans un nouvel espace? Quelles sont les mutations de l'espace physique qui auraient entraîné

1. Le sujet est défini comme un être construisant des agencements, soumis à la loi symbolique et contraint de passer par la parole pour s'établir lui-même dans sa vérité. Il est à chercher au carrefour de la connaissance de lui-même et de ce que les autres lui renvoient de cette présence au monde. Le concept de production de subjectivité développé par Félix Guattari, «De la production de subjectivité ", Revue Chimères, n 4 [en ligne], 1986 dernier accès avril 2011, disponible sur http://www.revue-chimeres.org/guattari/semin/semi.html, propose de voir le sujet collectif - la subjectivité - comme processus en lien avec les institutions qu'il nomme machines. En effet, les institutions ont produit et produisent encore des nouveaux types de subjectivité, le sujet ne se produit pas seul mais «sur des machines " au travers desquelles il construit des agencements. Ainsi, nous postulons dans cet article que les espaces du virtuel sont une nouvelle machine au service de la subjectivation.

2. Michel Foucault, Histoire de la sexualité III, Le souci de soi, Paris, Gallimard, 1984, p. 58,284 p. 
ce repli? Comment l'individu a-t-il conquis ou inventer un nouveau territoire interstitiel? Qu'est devenu l'espace de la chambre, lieu privilégié de la vie intime? Le virtuel n'est-il pas le devenir de la chambre?

Dans cet article nous souhaitons proposer une analyse de l'invention de ce nouvel espace à soi comme répondant à des questionnements sur les systèmes politiques en place. Intime, espaces du virtuel et régime politique seront vus ensemble afin de relever l'élaboration d'une nouvelle forme de subjectivité. Dans une première partie, nous nous attacherons à penser les espaces du virtuel comme l'invention d'un nouvel espace à soi, une forme émergente de la chambre. Puis, nous approfondirons la réflexion sur la manière dont ce nouvel espace s'imbrique dans les démocraties parlementaires.

\section{Invention d'un nouveau territoire du sujet : de la chambre aux espaces du virtuel}

Le 3 janvier 1983, le Times titre: "Les ordinateurs rentrent dans la maison $»^{3}$. L'espace de vie a dû trouver un lieu pour ce nouvel objet de la modernité. Il trônait dans la pièce principale des habitations dans les années 1990 et tout le monde le partageait. On le retrouve aujourd'hui dans les chambres des adolescents et pour l'adulte ou plutôt l'adulescent ${ }^{4}$ la portabilité de l'objet couplée à l'invention du wifi en fait un objet qui suit l'individu plus qu'un objet défini dans un espace particulier. On se retire aujourd'hui pour échanger des mails, surfer sur la vague des réseaux sociaux, faire des rencontres... Alain Corbin dit à ce sujet que : "Le désir d'un espace à soi n'a pas cessé de tarauder, notamment les jeunes. La tranquillité requise par la correspondance électronique et par la consultation des bases de données joue en faveur de la chambre [...]. $»^{5} \mathrm{Il}$ y a une forme d'intime qui se libère dans ces espaces alors qu'au siècle dernier l'intime existait uniquement dans l'espace de la chambre... Comme l'évoque Michelle Perrot, " on demeure dans la chambre » achetée chez "le vendeur de meubles, les femmes gardent la chambre" tout en lisant "leur livre de chevet». Elle est le lieu sacré de l'intimité... Les plaisirs du lit, de la rencontre des corps... L'intime aujourd'hui se diffuse dans les espaces du virtuel, il n'est plus circonscrit à l'espace de la chambre. Le virtuel est une chambre d'hôtel. En effet, pour Alain Corbin, la chambre d'hôtel a «des possibilités textuelles et scéniques infinies [...]

3. Otto Friedrich, "Computer moves in ", Times magazine "Machine of the year ", 3 novembre 1989.

4. Tony Anatrella, "Les «adulescents " ", Études 7/2003 (Tome 399), p. 37-47, 2003. Dernier accès avril 2011. Disponible sur : www.cairn.info/revue-etudes-2003-7-page-37.htm.

5. Alain Corbin "Toute l'intimité du monde ", La vie des idées.fr, 18 septembre 2009, p. 11. Dernier accès avril 2011. Disponible sur : http://www.laviedesidees.fr/Toute-l-intimitedu-monde.html. 
l'individu y éprouve une impression de suspens. La chambre d'hôtel suggère... l'attente de l'improbable rencontre... " Elle est un espace sans propriétaire, un espace de neutralité de l'identité où pourtant parfois se jouent des scènes très intimes : "On peut y jouir avec l'autre sans retenue et sans risque d'intrusion : parfois l'on y déprime... ou l'on s'y suicide. $»^{7}$ La chambre est avant tout un territoire. Mais, dans le cas de la chambre d'hôtel ce n'est pas « mon » territoire dans lequel je peux me déterritorialiser et m'y reterritorialiser en le retrouvant presque à l'identique... C'est un territoire qui n'est jamais complètement mien et jamais complètement autre. Quand je m'y territorialise, je me l'approprie pour m'en délester avec la même ardeur. Dans cet espace neutre, je dois me préciser. L'hypertexte dans le virtuel offre aussi une multitude de possibilités textuelles dans un lieu sans lieu où il est pourtant nécessaire que je m’inscrive rien que pour donner raison au dispositif. C'est une sorte de chambre d'hôtel multiforme. La constitution d'un profil sur les sites de rencontres se fait par la réponse à un questionnaire. Ces questionnaires, tous différents, permettent au sujet de se préciser comme on s'approprie l'espace physique de la chambre d'hôtel... Mais le sujet est totalement responsable de ce qu'il répond, peut choisir de mettre une photo de lui ou de détourner le dispositif en mettant une photo de Madonna. Il peut aussi choisir de ne pas répondre.

La chambre à soi est le lieu de l'intériorité. Dans Le petit Pierre, Anatole France écrit : "Dès que j'eus une chambre à moi, j'eus une vie intérieure [...]. Elle me séparait de l'univers, et j'y retrouvais l'univers. ${ }^{8}$ Ce qui en soi n'est qu'un fantasme de projection du territoire de l'être, la vie intérieure est inhérente à la vie... Cependant la chambre est liée à une représentation de l'espace de la vie intime. Elle est le lieu de la vulnérabilité due à l'état du sommeil, de la vie rêvée... Aujourd'hui que pouvons-nous dire de l'espace de la chambre? Que dire de la chambre des personnes qui vivent en colocation? Que penser de la réduction de l'espace de vie dans les villes : le studio? Dans la seconde moitié du $\mathrm{xx}^{\mathrm{e}}$ siècle, Michelle Perrot" parle d'un déclin du "culte caméral ». La chambre "impliquait un couple fondé sur la durée qui rêve d'enfants grandissant au sein de la cellule familiale ${ }^{10}$. La connotation sacrée disparaît, la chambre n'est plus le lieu de la naissance, ni de la mort. « L’ordre de la chambre reproduit l'ordre du monde "; en témoigne l'allégement intérieur de la première - celui du lit, des meubles, du décor - traduit l'affaiblissement

6. Ibid., p. 5.

7. Ibid., p. 5 .

8. Anatole France Le petit Pierre, Paris, Calmann-Lévy, 1921, p. 336.

9. Michelle Perrot, Histoire de chambres, Paris, Seuil, coll. "Librairie du Xxi siècle", 2009, 447 p.

10. Michelle Perrot, in Alain Corbin, op.cit., p. 11. 
de «sa densité existentielle ${ }^{11}$. Cet affaiblissement n'est-il pas le signe d'un renforcement existentiel par ailleurs, d'un déplacement dans un autre espace? L'invention du studio où la non-séparation des espaces ne permet plus le repli sur soi est-elle liée à la projection de l'intime dans le virtuel? Le virtuel serait-il le devenir de la chambre? Dans la colocation c'est autre chose, il y a des espaces partagés et des espaces privés. J'ai le souvenir d'un ami chez qui je résidais à Paris, qui, vivant en colocation, a fait quelque chose de très intéressant en termes de territoire. Trônait sur la table l'ordinateur portable connecté sur différents réseaux sociaux alors que nous prenions un verre... Après une discussion tardive, mon ami va se coucher, il me salue et part dans sa chambre avec son ordinateur sous le bras. La connexion wifi permet à tout moment de faire suivre l'espace du virtuel avec soi. Il faut, il me semble, se questionner sur les nouveaux rapports à l'espace depuis les objets portables et la connexion wifi. Ils permettent de ne plus être soumis à la "brutalité » de la déconnexion que l'on peut assimiler à une sorte de rupture du lien... Dans le cas de cet ami, l'espace intime virtuel se déplace jusqu'au plus intime et plus secret du lieu de la colocation : sa chambre. J'imagine qu'il n'a pas les mêmes échanges quand son ordinateur est dans la pièce de vie que quand il est dans sa chambre... La puissance du virtuel accrue par sa portabilité entre en résonance avec le lieu de l'actuel...

Alors s'il s'agit bien d'un nouvel espace à soi, ne faut-il pas en étudier les limites? Car un espace se définit par sa séparation avec les autres espaces. Qu'est-ce qui délimite l'espace dans d'un point de vue physique? Pour Didier Anzieu $^{12}$, la peau est ce qui me permet de faire contact avec le monde et aussi ce qui me permet de prendre conscience d'où je me situe. Elle me contient et me différencie en même temps. Surface d'étayage aux représentations, à la construction de l'espace psychique symbolique, c'est de cette réflexivité que se construit le schéma corporel. La peau véritable réceptacle de l'expérience du toucher de l'effleurement, ou de la sensation d'un autre corps à distance est une frontière essentielle dans l'espace physique. Mais alors dans ce nouvel espace de la Toile où est la limite, la frontière? Existe-t-elle? Quelle est la peau du virtuel?

Dans son chapitre sur La parole du seuil Jean-Claude Reinhardt évoque la part importante de la formulette qui se répète notamment dans les contes après chaque événement que vit le héros. "La formulette est liée [...] à tous « les lieux obligés » que le héros doit traverser : «zones neutres » entre deux

11. Ibid.

12. Didier Anzieu, Le moi-peau, Paris, Dunod, 1985, 291 p. 
territoires [...]. ${ }^{13}$ Sur les sites de flux vidéo, Anne Cauquelin (2003) ${ }^{14}$ explique qu'il est simple de se connecter à ces intimes de vie : il suffit d'avoir les adresses des personnes connectées. Autant parler de formulette alors. Pour aller sur ma page Facebook ou Twitter, j'ai un " nom d'utilisateur » et un " mot de passe ». Tout autant de formulettes, de zones neutres où je reste au seuil et finalement de non-territoire en non-territoire, d'utopie en utopie. Il n'est pas du tout simple de rentrer dans l'espace virtuel de quelqu'un comme propose de le penser Anne Cauquelin. Ce que je veux souligner avec l'idée du seuil c'est que la barrière de l'intime n'est pas inexistante, n'est pas absente, ni même poreuse, elle a une épaisseur qui ne peut être ôtée sauf si on connaît l'adresse, la formulette et qu'on passe l'étape du judas. Les internautes clouent des sacra sur leur seuil, usent de protections diverses avec autant de sonnettes, de formulettes et de judas que possible. Le pseudonyme, la fausse photo, le contrôle des accès à certains contacts sont là pour révéler que nous sommes dans l'espace d'un seuil, une zone entre l'extérieur et l'intérieur. On observe comment le désir de cacher prend forme dans la délimitation de son espace. La créativité des internautes pour construire des barrières met en évidence la conquête d'un territoire privé ou en tout cas le désir de le conquérir. Mais de quoi nous protégeons-nous dans ces espaces par ces multiples sacra? Nous souhaitons tout autant être vus que ne pas l'être... Il y a comme un paradoxe insoluble dans le souhait de faire un espace à soi dans un espace surpeuplé "d'autres". Peut-être parce que ce territoire est conquis comme un espace à l'interstice du sujet et des autres. Événement de l'altérité, pour Gilles Deleuze le virtuel est un territoire étranger à l'intérieur ${ }^{15}$, la potentialité de rencontrer l'autre et l'autre en soi. L'être présent dans son invisibilité et visible dans son absence devient insaisissable, car dans l'intervalle. Les espaces virtuels seraient les territoires de la vacuité du sujet contemporain entre l'intime de l'être et sa monstration.

Pierre Lévy insiste sur l'idée que le virtuel " n'est bien souvent pas là ${ }^{16}$. Où est le lieu de la conversation téléphonique? Où est le lieu des échanges

13. Jean-Claude Reinhardt, Jean Bouisson, Seuils, parcours, vieillissements, Paris, L'Harmattan, 2000, p. 27, 241 p.

14. Anne Cauquelin, L'exposition de soi, du journal intime aux Webcams, Paris, Éditions Eshel, 2003, 95 p.

15. Expression faisant référence au concept de unheimlich, de Sigmund Freud (Sigmund Freud, L'inquiétante étrangeté, Essais de psychanalyse appliquée, Paris, Gallimard, coll. "Idées ", 1919, 1976, 378 p.). L'inquiétante étrangeté, sa traduction française, est l'expérience d'un être étranger à l'intérieur de soi, c'est un double de soi étranger à soi-même né du travail de l'inconscient dans le refoulement.

16. Pierre Lévy, Qu’est-ce que le virtuel, Paris, La découverte/Poche, Essais, 1998, p. 17, $153 \mathrm{p}$. 
numériques? Des lieux inassignables qui nous font quitter le « là. » Cependant, le produit de cet événement est entre des choses clairement situées. «Lorsqu'une personne, une collectivité, un acte, une information se virtualisent, ils se mettent "hors-là ", ils se déterritorialisent. " ${ }^{17}$ Ces communautés virtuelles mettent en lumière les modalités de l'être ensemble : la constitution d'un nous déterritorialisé. Le territoire défini de l'être se libère de ses anciennes définitions. Mais alors, dans ce double mouvement décrit par Gilles Deleuze et Félix Guattari, les individus se déterritorialisent pour se reterritorialiser et réaffecter leur territoire : ne peut-on pas penser que les sujets désirent ne plus être dans aucun territoire, ne plus être limités par l'espace physique, mais demeurer dans une béance entre déterritorialisation et une territorialisation sans territoire ou que la communauté, ce nous virtuel fonde un nouveau territoire symbolique de l'être? Pourquoi ne pas parler de territorialisation de nouveau territoire créé par le sujet? Car ces deux auteurs nous ont déjà avertis que " [...] chacun, à tout âge se cherche un territoire, supporte ou mène des déterritorialisations, et se reterritorialise presque sur n'importe quoi, souvenir, fétiche, ou rêve [...] ${ }^{18}$ alors pourquoi pas sur le virtuel?

\section{Diffusion de l'intime comme évolution du bio-pouvoir dans les démocraties parlementaires}

L'arrivée de l'ordinateur dans l'espace des habitations a transformé notre rapport à l'espace dans nos vies intimes. Cet espace est borné par des limites d'accès comme tout territoire permettant la subjectivation de l'individu. Mais alors, pourquoi le sujet a-t-il besoin aujourd'hui de ce nouveau territoire pour se subjectiver? Quel rapport ces discours de soi entretiennent-ils avec le pouvoir démocratique et avec la notion foucaldienne de bio-pouvoir ${ }^{19}$ ? Ce pouvoir de voir et d'être vu dans sa vie intime donne-t-il une légitimité à nos existences?

La constitution de profil dans les sites de dating, de matchmaking par exemple renvoie à la notion de persona... L'idée du masque, du double de l'avatar. Mais un double de duplication qui comme l'entend Clément Rosset ${ }^{20}$ ne fait que signifier ce qu'il copie pour le rendre plus perceptible, plus visible... "Si je suis perçue, vue par le prisme de mon double de duplication c'est que j'existe ". Â la différence du double de remplacement qui tue ce qu'il copie... Comme dans l'Antiquité, la persona doit être validée par la communauté. « La

17. Ibid., p. 18.

18. Gilles Deleuze, Felix Guattari, Qu'est-ce que la philosophie? Paris, Les Éditions de Minuit, 1991, p. 66, 206 p.

19. Michel Foucault, La volonté de savoir, Histoire de la sexualité, t. I, Paris, Gallimard, 1976, 248 p.

20. Clément Rosset, Fantasmagories, Paris, Éditions de Minuit, 2006, 108 p. 
lutte pour la reconnaissance est donc à chaque fois, une lutte pour le masque, mais ce masque coïncide avec la personnalité que la société reconnaît à chaque individu. ${ }^{21} \mathrm{Si}$ mon intime est vu alors j'existe. Et si en retour je peux voir l'intime des autres c'est qu'ils existent. La rencontre des écritures de l'intime légitime nos existences.

Faisons un retour sur l'étymologie du mot intime afin de préciser clairement comment nous l'entendons. L'intime en tant que substantif et non pas en tant qu'adjectif, provenant du latin intimus, superlatif de la forme archaïque de intus en dedans : "in " signifiant dans et "tus" ayant une valeur locative. C'est-à-dire que le "dans " a un locus, un lieu, un territoire à l'intérieur de lui-même. Aujourd'hui, l'intime cherche un territoire à l'extérieur. Avec la religion, il a trouvé une place dans la confession; avec la psychanalyse dans la séance. Mais dans ces cas, les territoires ne sont pas publics, l'homme de foi ou le psychanalyste sont tenus par le secret professionnel. Lintime semble vouloir maintenant un lieu sorti du secret tout en restant protégé par les sacra du seuil.

De plus, l'intime ne reste plus une affaire privée. Il se perçoit facilement dans l'espace public. On peut être le spectateur de moments intimes qu'un couple partage dans la ville et l'auditeur de conversations téléphoniques intimes dans les transports en commun. Loin encore une fois de condamner ces usages, il est plus riche de comprendre cette évolution du concept du bio-pouvoir dans les démocraties parlementaires. Ce serait bien plus simple de cliver les espaces pour penser un concept, mais la réalité du monde des possibles révélée par la toile oblige à penser le territoire de l'intime dans un espace d'enchevêtrement entre le virtuel et l'actuel ${ }^{22}$. Mais quel rapport ces nouveaux territoires de l'intime ont-ils avec la démocratie?

La vie intime qui peut se lire, se voir et s'entendre sur la toile fait éclater la distinction entre espace public et espace privé. Dans son ouvrage La privatisation de l'intime, Michael Foessel, philosophe spécialiste en philosophie politique notamment, se demande: "Qu'est-ce que l'intime pour que la

21. Giorgio Agamben, Nudités, traduit de l'italien par Martin Rueff, Paris, Rivages, 2009, p. 82,191 p.

22. Le virtuel ne s'oppose pas au réel mais à l'actuel. Le virtuel n'a que peu de lien avec le réel, l'illusoire ou l'imaginaire. Il est puissance avant tout. Virtualis en latin issu de virtus force, puissance est l'origine étymologique de ce mot. L'arbre est présent dans la graine en puissance. L'actuel c'est l'arbre en train de pousser. "L'actuel ne ressemble en rien au virtuel : il lui répond ", op. cit, p. 15. Ce qui est acte s'oppose à ce qui est en puissance. Dans l'usage de ces dispositifs, l'individu entre actuel et virtuel montre ce qu'il est en train de devenir (la virtualité de l'être) pour affirmer son essence (actualité de l'être). Pierre Lévy pose la virtualisation comme dynamique et non plus le virtuel comme manière d'être. Pour lui, la virtualisation est synonyme d'exode. "L'actualisation allait d'un problème à une solution. La virtualisation passe d'une solution donnée à un autre problème. ", op. cit., p. 16. 
démocratie se trouve fragilisée par son dévoiement. ${ }^{23} \mathrm{Il}$ évoque la manière dont les hommes politiques ont recours à l'exposition de leur vie intime, il parle même de "pipolisation de la politique ". " Il reste alors à comprendre dans quelle mesure une certaine idée de l'intime est indissociable des promesses démocratiques, au point que son discrédit affecte le rapport que nous entretenons avec la politique. ${ }^{24}$ Et plus que de la pipolisation de la politique c'est de la pipolisation de l'intime dont il est question. Pour Michael Foessel l'intime est la marque d'une proximité incomparable avec dieu et le définit comme concept relationnel. "Abordé sans recours à la transcendance, l'intime conserve cette dimension dialogique : nos sentiments "intérieurs" sont des rapports avec les autres et non des propriétés d'un Moi solitaire. $»^{25}$ Pour lui, nous ferions la confusion entre l'intime et espace privé : "Nous montrerons que non seulement l'intime n'est pas le privé mais qu'il est interdit de réduire l'expérience humaine à la dualité public-privé. ${ }^{26}$ Le propos de Michael Foessel n'est pas nouveau déjà Michel Foucault ${ }^{27}$ dans ses travaux sur le bio-pouvoir expliquait cette implication croissante de la vie naturelle du sujet dans le pouvoir. Le droit à la vie, au corps, à la santé, au bonheur; en somme le Droit dans ce qu'il renvoie de fantasmatique sur la libération de toutes les oppressions constitue cette nouvelle forme de pouvoir ${ }^{28}$. On assisterait à une totale politisation de la vie. Giorgio Agamben ${ }^{29}$ (1997) dans Homo Sacer I cite Karl Löwith : « [...] Depuis l'émancipation du Tiers État, la formation de la démocratie bourgeoise et sa transformation en une démocratie industrielle de masse, et jusqu'au moment décisif où le phénomène s'est inversé : il aboutit aujourd'hui à une totale politisation de tout y compris de certaines sphères de la vie apparemment neutres. $»^{30}$ L'intime, la sphère entre-deux, entre espace public et espace privé prend sa place en politique. Giorgio Agamben le souligne dans son chapitre, La politisation de la vie. L'individu croit gagner des espaces de liberté après les affrontements avec le pouvoir avec comme effet pervers une

23. Michael Foessel, La privation de l'intime, Paris, Seuil, 2008, p. 8, 157 p.

24. Ibid., p. 11.

25. Ibid., p. 13.

26. Ibid., p. 15.

27. Michel Foucault, La volonté de savoir, Histoire de la sexualité, t. I, Paris, Gallimard, 1976, 284 p.

28. Ibid.

29. Dans Homo Sacer I, Giorgio Agamben reprend le concept foucaldien de bio-pouvoir en le faisant évoluer vers le pouvoir souverain. Il analyse l'origine du pouvoir où la vie fait l'objet d'une décision souveraine alors que pour Michel Foucault le bio-pouvoir échappe au pouvoir souverain.

30. Karl Löwith, in Giorgio Agamben, Homo Sacer I, le pouvoir souverain et la vie nue, Paris, Seuil, 1997, p. 131, 213 p. 
inscription plus profonde de leur vie dans l'espace public: offrir une assise nouvelle et plus terrible au pouvoir souverain dont il voudrait s'affranchir.

Selon Giorgio Agamben, les démocraties parlementaires se seraient même transformées en états totalitaires et inversement par le glissement de la politique vers la bio-politique afin de créer : «Une forme d'organisation politique la plus efficace pour garantir le contrôle, la jouissance et le souci de la vie nue. ${ }^{31}$ Il n'y a plus de distinction politique claire entre partis par exemple car c'est la vie nue qui est le point d'ancrage de la politique. On apprend la nouvelle relation du président de la république à Disneyland, la séparation de François Hollande et Ségolène Royal qui répondent de leur vie intime sur différentes émissions télévisuelles ou radiophoniques. Et plus récemment c'est Anne Sinclair qui annonce la candidature à la présidence de son mari en disant : qu'elle ne souhaite pas qu'il «fasse un second mandat à la tête du $F M I »^{32}$ où on découvre une épouse parlant de l'ambition qu'elle a pour son époux plutôt que d'orientation politique. Le 14 avril dernier, lors de la matinale de France Inter avec Bruno Duvic, Nicolas Hulot en appelle à un « je souhaite toujours avoir un peu d'espace pour mon incohérence ${ }^{33}$ quand une question polémique d'un auditeur lui est posée sur sa posture d'écologiste alors que sa fondation Ushuaia reçoit le financement du "plus grand pollueur de la planète : Rhône-Poulenc $»^{34}$. La question concerne pourtant bien la cité mais Nicolas Hulot nous parle de son intériorité...

Dans l'intime il est toujours question des origines du sujet, quête sans cesse relayée par les discours du religieux, du médical et des séries policières car comme le dit Giorgio Agamben : " [...] le souverain agit de plus en plus en symbiose non seulement avec le juriste mais aussi avec les médecins, le savant, l'expert et le prêtre $»^{35}$. Le dossier médical numérique qui nous suit avec notre carte vitale est une belle illustration de ce propos. Finalement, l'exposition de la vie nue dans l'espace public aurait conduit l'intime à se déjouer du politique (ce n'est peut-être qu'une illusion) pour se retrouver dans l'espace du virtuel : "C'est mon droit après tout de faire ce que je veux avec mon image. " Nous voyons comment il est d'ailleurs difficile pour le législateur de légiférer sur ces espaces. L'effet pervers serait la dilution de l'intime dans la politique, qu'elle s'empare des écritures dans le virtuel. Les

31. Giorgio Agamben, Homo Sacer I, le pouvoir souverain et la vie nue, Paris, Seuil, 1997, p. 132,213 p.

32. Le point, 9 février 2011.

33. Patrick Cohen, 7/9 de France Inter, 14 avril 2011 [en ligne]. Dernier accès avril 2011. Disponible sur : http://www.dailymotion.com/video/xi7t7g_nicolas-hulot_news.

34. Ibid.

35. Op. cit., p. 132. 
derniers exemples de personnes condamnées pour des propos diffamatoires sur des réseaux sociaux illustrent bien cette dérive. Pourtant quand je suis chez moi entre amis nous partageons des propos de ce genre. Il y a comme une indifférenciation entre la zoé et le bios. Le problème n'est pas que la zoé entre dans la polis, mais plutôt que "l'espace de la vie nue [...] finit (sic) par coïncider avec l'espace politique $»^{36}$. L'intime est au cœur de ce jeu des espaces : il est le jouet du politique.

Pour Giorgio Agamben, la démocratisation moderne cherche constamment à transformer la vie nue elle-même en une forme de vie afin de trouver pour ainsi dire, le bios de la zoé. Les écritures de l'intime illustrent bien le propos d'Aristote sur le fait que ce qui différencie l'Homme de l'animal ce n'est pas la voix mais le discours car, il permet de dire le bien, le mal, le juste, l'injuste et : "c'est la communauté des sentiments qui engendre famille et cité ${ }^{37}$. Peut-on penser que l'intime faisait partie de la zoé et qu'il tend aujourd'hui à faire partie du bios? Ou l'intime a-t-il toujours été dans l'entre-deux, entre vivre comme tous - nous avons tous une vie intime - et vivre en tant qu'être singulier - nous avons tous une manière propre de le vivre cette vie intime. La forme de l'intime contenue dans le bios s'écrirait sur la toile alors que la zoé quant à elle se projetterait à travers la barrière poreuse (avec le bios) par petites touches pour finalement demeurer dans sa propre sphère. Cette hypertextualité du sujet serait-elle l'évolution des démocraties modernes? L'intime est-il l'élément qui fait le lien entre bios et zoé? Possède-t-il deux formes distinctes relatives à ces deux formes de vie? Est-il aujourd'hui le concept interstitiel de la vie?

Le sujet use-t-il de ce nouvel espace dont il définit sans cesse les frontières pour participer à sa subjectivation rendue de plus en plus difficile dans une société démocratique où tout est encore contrôlé? Ce territoire n'est-il pas le lieu par excellence de son devenir sujet?

Finalement, dans un mouvement incessant de l'espace diffus d'actuel au virtuel d'étranges ballets entre communication orale et écrite de l'intime ouvre une nouvelle voie de subjectivation à l'Homme donnant naissance à " une subjectivité machinique d'un nouveau genre " ${ }^{38}$. Pour Félix Guattari,

36. Op. cit., p. 17.

37. Aristote, Politique, 1253a 10-18.

38. Félix Guattari, "De la production de subjectivité ", Revue Chimères, n 4 [en ligne], 1986, dernier accès avril 2011. Disponible sur http:/www.revue-chimeres.org/guattari/semin/semi.html, p. 13. L'arrivée de l'ordinateur est pour Felix Guattari un bouleversement pour la production de subjectivité (i.e. comment devient-on sujet) : « [...] les contenus de la subjectivité dépendent toujours plus d'une multitude de systèmes machines. Aucun domaine d'opinion, de pensée, d'images, d'affects, de narrativité ne peut désormais prétendre échapper à l'emprise envahissante de «l'assistance par ordinateur »." (Ibid., p. 1). 
il n'y a pas de sujet mais un sujet collectif dont le territoire est la planète. "La subjectivité est plurielle et polyphonique. " ${ }^{39}$ Le virtuel jusqu’à Google Earth offre la possibilité d'appréhender ce territoire dans son ensemble. Il offre le monde "en puissance ". Ce sujet collectif qui s'oppose au sujet unique, Félix Guattari le nomme : subjectivité. La subjectivité à travers le virtuel rencontre différentes "machines " : la machine de l'écriture de soi, la machine économique, la machine de la morale, la machine désirante... C'est comme si dans le virtuel, la subjectivité rencontrait les mêmes machines contenues dans l'actuel mais cette fois-ci en puissance dans un espace dont les limites sont toujours à définir et où les sociétés démocratiques n'ont pas encore trouvé à le contraindre. Autant d'agencements machiniques pour créer la subjectivité.

Lélévation du niveau de vie en a transformé son espace. En effet, de la chambre collective partagée par la famille à la disparition du lit commun au profit de la chambre à soi c'est tout un territoire du secret et de l'intime qui se trouve créé. À ce même moment, on ne parle plus de l'Homme mais du sujet; de celui qui a conscience d'être au monde. De cette chambre individuelle au siècle dernier à l'espace à soi dans le virtuel délimité par des frontières que le sujet renouvelle sans cesse dans chaque dispositif, dans cet enchevêtrement des espaces public et privé, la vie nue et l'intime s'offrent à la vue des sociétés démocratiques mais dans un espace qui pour le moment est en deçà du droit pour trouver sa subjectivité. Cet espace inventé par le sujet fonde un territoire d'expression de soi. L'intime, concept interstitiel entre bios et zoé évolue dans un espace lui aussi interstitiel entre public et privé. Le virtuel se présente comme une nouvelle machine qui produit une forme émergente de subjectivité. Pour Michel Foucault ${ }^{40}$, le souci de soi serait survenu à la suite d'un affaiblissement du cadre politique des cités grecques. La pipolisation de la vie politique, forme d'affaiblissement est-elle la cause de ce retour sur soi? Cette diffusion dans ces espaces va-t-elle augmenter le pouvoir des états pour en faire des états totalitaires encore plus puissants? Éric Schmidt, PDG de Google (2010) dit quelque chose de terrifiant lors de la Conference Techonomy : "Avec 14 photos de vous, nous avons la capacité de vous identifier, vous croyez qu'il n'y a pas 14 photos de vous sur la toile? Il y a des photos de Facebook. $»^{41}$ Mais de quelle identité parlons-nous? Ou s'agit-il d'un contre-pouvoir où l'individu se saisit de ces nouveaux espaces pour former une nouvelle société?

39. Félix Guattari Chaosmose, Paris, Galilée, 1992, 186 p.

40. Michel Foucault, Histoire de la sexualité III, Le souci de soi, Paris, Gallimard, 1984.

41. Eric Schmidt, "Conference Techonomy", in Philippe Rivière, "Facebook miroir magique ", Le Monde Diplomatique, décembre 2010. 


\section{Bibliographie}

Giorgio Agamben, Homo Sacer I, le pouvoir souverain et la vie nue, Paris, Seuil, 1997, 213 p.

p. 82,191 p.

, Nudités, traduit de l'italien par Martin Rueff, Paris, Rivages, 2009,

Tony Anatrella, «Les « adulescents " ", Études 7/2003 (T. 399), p. 37-47, 2003. Dernier accès avril 2011. Disponible sur : www.cairn.info/revue-etudes2003-7-page-37.htm.

Didier Anzieu, Le moi-peau, Paris, Dunod, 1985, 291p.

Aristote, Politique, 1253a 10-18.

Anne Cauquelin, L'exposition de soi, du journal intime aux Webcams, Paris, Éditions Eshel, 2003, 95 p.

Alain Corbin "Toute l'intimité du monde», La vie des idées.fr, 18 septembre 2009, p. 11. Dernier accès avril 2011. Disponible sur : http://www. laviedesidees.fr/Toute-l-intimite-du-monde.html.

Gilles Deleuze, Felix Guattari, Qu'est-ce que la philosophie? Paris, Les éditions de minuit, 1991, 206 p.

Michael Foessel, La privation de l'intime, Paris, Seuil, 2008, 157 p.

Michel Foucault, Histoire de la sexualité III, Le souci de soi, Paris, Gallimard, 1984, 284 p.

_- La volonté de savoir, Histoire de la sexualité, T. I, Paris, Gallimard, 1976, 248 p.

Anatole France Le petit Pierre, Paris, Calmann-Lévy, 1921, 336 p.

Sigmund Freud, Linquiétante étrangeté, Essais de psychanalyse appliquée, Paris, Gallimard, coll. "Idées », 1919, 1976, 378 p.

Otto Friedrich, "Computer moves in ", Times magazine "Machine of the year ", 3 novembre 1989.

Félix Guattari Chaosmose, Paris, Galilée, 1992, 186p.

—, "De la production de subjectivité », Revue Chimères, n 4 [en ligne], 1986, dernier accès avril 2011. Disponible sur http://www.revue-chimeres. org/guattari/semin/semi.html.

Pierre Lévy, Qu'est-ce que le virtuel, Paris, La découverte/Poche, Essais, 1998, p. 17,153 p.

Michelle Perrot, Histoire de chambres, Paris, Seuil, coll. "Librairie du XXI siècle ", 2009, 447 p.

Jean-Claude Reinhardt, Jean Bouisson, Seuils, parcours, vieillissements, Paris, L'Harmattan, 2000, $241 \mathrm{p}$.

Éric Schmidt, "Conference Techonomy ", in Philippe Rivière, "Facebook miroir magique », Le Monde Diplomatique, décembre 2010.

Clément Rosset, Fantasmagories, Paris, Éditions de Minuit, 2006, 108 p. 
Invention d'un nouveau territoire de subjectivation...

\section{Source vidéo :}

Patrick Cohen, 7/9 de France Inter, 14 avril 2011 [en ligne], dernier accès avril 2011. Disponible sur: http://www.dailymotion.com/video/xi7t7g nicolas-hulot_news 